\title{
An examination of college mathematics majors' understandings of their own written definitions
}

\section{Authors: David S. Dickerson \& Damian J. Pitman}

NOTICE: this is the author's version of a work that was accepted for publication in Journal of Mathematical Behavior. Changes resulting from the publishing process, such as peer review, editing, corrections, structural formatting, and other quality control mechanisms may not be reflected in this document. Changes may have been made to this work since it was submitted for publication. A definitive version was subsequently published in The Journal of Mathematical Behavior [VOL\# 41, (March 2016)] DOI\# 10.1016/j.jmathb.2015.11.001

Dickerson, David S. , and Damien J. Pitman. "An examination of college mathematics majors' understandings of their own written definitions." The Journal of Mathematical Behavior 41 (March 2016): 1-9. DOI: 10.1016/j.jmathb.2015.11.001

Made available through Montana State University's ScholarWorks scholarworks. montana.edu 


\title{
An examination of college mathematics majors' understandings of their own written definitions
}

\author{
David S. Dickerson ${ }^{\mathrm{a}, *}$, Damien J. Pitman ${ }^{\mathrm{b}}$ \\ a SUNY Cortland, Cortland, NY 13045, USA \\ b Montana State University, Bozeman, MT 59717, USA
}

\begin{abstract}
This qualitative study of ten undergraduate mathematics majors examined students' abilities to write definitions and found that students at the advanced level of undergraduate mathematical study have difficulty creating definitions that conform to their concept images or to accepted definitions of basic concepts. This is due in part to (1) failure to consider key examples when writing definitions, (2) weak concept images for the concept in question, and (3) vague concept images for related concepts. The results of this study have implications for secondary-level and college-level mathematics instruction.
\end{abstract}

\section{Introduction and framework}

Mathematical proofs have long been known to be difficult for students of mathematics. Many misconceptions have been identified as well as many areas of difficulty. Moore (1994) listed seven possible reasons for students' inability to write proofs and most had something to do with either mathematical language or definitions. Vinner (1977) suggests that students do not know how to use definitions in proofs. This may be because students must first fully understand definitions before they can use them. In this study, we wanted to investigate students' use of definitions that they understood. We did this by first asking them to provide for us their own definitions of familiar concepts, and then asking them to use those definitions to make determinations. What resulted was a cycle of refinement of definitions in which participants attempted to bring their definitions into closer agreement with their concept images.

\subsection{The nature of mathematical definitions}

Definitions play a central role in mathematics. Mathematicians and students of mathematics use definitions routinely but seldom think about the nature of mathematical definition (Wilson, 1990). One use for a mathematical definition is really as a mathematical short-hand or abbreviation. We define a function $f: D \rightarrow \mathbb{R}$ to be continuous at some $c$ in $D$ provided for every $\varepsilon>0$ there is a $\delta>0$ such that if $|x-c|<\delta$ then $|f(x)-f(c)|<\varepsilon$. From that moment on, we do not have to refer to such functions as "functions with the property that for every $\varepsilon>0$ there is a $\delta>0$ such that..." We just call such functions "continuous." The process of defining in mathematics is merely the creating of names to go with concepts-typically useful or interesting concepts. 
Definitions in mathematics are somewhat different than they are in natural language in that they are stipulative rather than extracted. "Extracted definitions report usage, while stipulative definitions create usage" (Edwards \& Ward, 2004, p. 412). New words emerge all the time by common usage and extracted definitions describe that usage. Extracted definitions describe how a word is used and what is meant by those who use it. By contrast, mathematical definitions are stipulative. They are created on the advice of experts and prescribe how a word shall be used and what it shall mean.

Beyond being created on the advice of experts, mathematical definitions need no further justification. They can neither be proven nor disproven, only accepted or rejected. Consider the following commonly accepted definition: A quadrilateral is called a trapezoid provided it has at least one pair of parallel sides. Under this definition, a square would be a trapezoid. If we are satisfied that a square should be considered to be a trapezoid, then we can accept this definition. If not, we can reject it in favor of one that disqualifies squares from consideration.

\subsection{Students' use of mathematical definitions}

To have at least a partial understanding of a concept means to have an image of it. The term concept image is used "to describe the total cognitive structure that is associated with the concept, which includes all the mental pictures and associated properties and processes" (Tall \& Vinner, 1981, p. 152). Thus, being able to recite a definition of a concept from rote memory does not guarantee a robust understanding of the concept (Vinner, 1991). Tall and Vinner (1981) report that students' concept images may be quite different from the definitions of those concepts. Thus, when students write proofs from faulty or incomplete concept images, even a correct line of reasoning can lead one astray.

Moore (1994) identified several difficulties students have in writing mathematical proofs including: students often (1) do not know the definitions, (2) have little intuitive understanding of the concepts in question, (3) have concept images that are inadequate for doing proofs, (4) do not generate and use their own examples, (5) do not know how to use definitions to obtain the overall structure of a proofs, (6) neither use nor understand mathematical language, and (7) do not know how to begin a proof. All but the last of these has something to do either with definitions, or with mathematical language. This list is by no means exhaustive. Perhaps most notably absent from the above list is students' difficulties with the underlying logic of proofs. Harel and Sowder (1998) observed that while students in their study attempted to prove theorems by example (often a single example), they seldom attempted to refute them by counterexample and were generally unconvinced by disproofs of this method. More recently, in working with 23 students of advanced undergraduate mathematics, Wheeler and Champion (2013) identified several errors and misconceptions that students displayed when writing proofs. Among the difficulties concerning the underlying logic of proofs were (1) beginning with the conclusion, (2) interfering knowledge (modeling an onto proof after a one-to-one proof), and (3) proof by example.

Edwards and Ward (2004) reported that students do not use mathematical definitions in the same ways that mathematicians do. Even students who can state and explain definitions often reason from their concept images. When concept images and concept definitions conflicted for two of their participants, they apparently believed that the definition had not been correctly extracted and argued incorrectly from their concept images instead. Dickerson and Pitman (2012) reported that reasoning entirely from incomplete concept images was common among college mathematics majors even when the definitions were given, and rejecting the given definitions in favor of vague concept images was common. In contrast, while mathematicians also reason from their concept images, the reasoning given in formal proofs is based on definitions. Moreover, if confronted with difficulties while reasoning from their concept image, mathematicians defer to the definition.

\subsection{Methods}

The questions that guided our study are: (1) What difficulties do students encounter when attempting to write definitions to match their concept images? and (2) How do students use definitions of their own creation? Ten college mathematics majors participated in this study. Each was currently a student in an upper division course in college geometry or algebraic structures. Participants filled out a paper survey and each was interviewed twice by the authors. The data for this study comes from the survey and the interviews.

In the survey, participants were asked to define the terms even, prime, square, parallel lines, tangent line, and trapezoid, as carefully as they could. We felt that college mathematics majors would have fairly robust concept images of these middle school and high school level concepts and would be more successful at writing definitions for them than they would be for concepts such as ring or uniform continuity. It is important to note that some of these terms are defined differently from author to author. For example, prime is defined by some in such a way that allows for negative numbers to be prime. Our intent was not to determine whether our participants' concept images would allow primes to be negative, squares to be trapezoids, or zero to be even; but whether they could write definitions consistent with their concept images and then use their written definitions to make determinations of examples and non-examples. After reading each survey, the two authors met to create follow-up questions to ask each participant as the basis for the first of two semi-structured interviews.

The interviews were semi-structured, audio-recorded, and transcribed. The interview was task-based (Goldin, 2000) in which we asked participants to re-read the definitions they provided on the survey and then to respond to approximately three or four follow-up questions for up to five of their definitions to probe their understanding. Our follow-up questions were meant to determine whether the written definition really captured the participant's concept image, and if not to provide an opportunity for the participant to amend their definition to better describe their concept image. For example, 
one participant Maria defined a trapezoid as "A shape with 2 sides that are parallel." Our follow-up questions were, "A regular hexagon has 3 pairs of parallel sides; is a regular hexagon a trapezoid?" and "A parallelogram has two pairs of parallel sides; is a parallelogram a trapezoid?" Our intent was to see if students could correctly apply their own written definitions to make these determinations and whether they could make modifications to their definition consistent with their concept images. Participants were encouraged to rewrite their definitions in any way they felt appropriate and as often as they felt it necessary, and these artifacts were collected and used as data in this study.

Asking our participants to write, re-read, and revise definitions is mathematical in that we were asking our participants to behave in ways that a mathematician might. A mathematician might have an image for a concept such as Cauchy sequence or pseudoprime number and then write a definition for that concept. She might go through a series of refinements of both her definition and her concept image until she arrives at a definition that she will treat as stipulative and accept the consequences of further tests of examples. At the beginning of this process, she extracts the meaning of the term from her concept image by attempting to describe what she means when she uses that term. But by the end, she stipulates a definition that prescribes what that term means and how it shall be used.

After the interviews were all completed, the two authors met to discuss the coding of the data. Once we agreed on a set of codes, each author coded each interview independently and then we met to discuss our coding of each interview. When there were differences, we discussed them with each other until they were resolved. For example, the code Failure in Applying Definition described transcripts in which participants applied only part of the definition, or avoided referencing the definition, or did not accept or recognize the consequences of a definition whereas the code Success in Applying Definition described transcripts in which the participant accepted the consequences of a definition-even a faulty definition.

\section{Results}

Before describing our results, it might be of value to say that virtually none of our participants' initial written definitions were correct in that they were not definitions that would be accepted by the mathematical community. During the course of our interviews, every participant revised nearly every one of their initial definitions until either they were satisfied that it adequately described their concept image, or they gave up for a variety of reasons. It might also be of value to reveal that more than half of our participants' revised definitions were still not correct. With this stated, we will not be evaluating the correctness of their definitions, rather we will be trying to evaluate how successfully our participants wrote definitions that described their concept images, and how they used the definitions of their own creation.

\subsection{Writing and revising definitions}

In this study, we asked our participants for their own definitions. Our thinking was that if the participants supplied their own definitions, they would understand those definitions, and hence be more successful at applying those definitions. In this study, participants' initial, written definitions were typically weak descriptions of their own concept images but over the course of our conversations where we supplied examples and non-examples, participants' definitions were often refined and brought into closer alignment with their own concept images.

\subsubsection{Successful use of mathematical reasoning}

We asked eight of our ten participants if zero was even and all argued correctly from their initial written definitions that zero was even, however four of them subsequently amended their definition to specifically exclude zero. Colin refined his initial definition from "Any number divisible by 2," to "Excluding zero, an even number is a number that can be written as $2 c, c$ is an integer." Similarly, Tally changed her definition from "A rational number $n .$. " to "Any non-zero rational number $m$..." The end result of both of these refinements reflects correct mathematical reasoning to bring a concept definition into closer agreement with a concept image that excludes zero from the set of even numbers.

We asked six of our participants if 1 was a prime number and all argued correctly from their own initial written definitions that either it was or it wasn't. Faye amended her initial written definition from, "A prime number is a number that is only divisible by itself and 1" to "A prime number is a positive integer whose only positive divisors are one and itself, not including 1." This change in language was to rule out two cases. First, she said "I know I meant that. . 1 is not a prime number," and second, she decided that by her initial definition, 7 could not be prime because it was divisible by \pm 1 and \pm 7 , so she amended her definition to take care of these perceived inadequacies and in so doing brought her final written definition into closer agreement with her concept image.

The above cases are typical of our data in and support our conjecture that participants' initial definitions are poor reflections of their own concept images. This is apparent given the large number of mismatches between initial and final written definitions. In the majority of cases, the participants in this study either revised or abandoned their initial written definitions in part because they (1) had not considered key examples and non-examples, (2) had weak concept images of the concept in question, or (3) had vague concept images for related concepts. We provided examples and non-examples to see if they could correctly argue from their definitions to make these determinations and to amend their definitions if necessary to bring them into closer agreement with their concept images. The participants were typically proficient in applying their definitions to previously unconsidered cases. Moreover, upon recognizing mismatches between their written definitions 
and their concept images, participants were usually successful at amending their definitions to more accurately reflect their concept images.

\subsubsection{Failure to consider key examples}

All of our participants failed to consider key examples and non-examples in writing most of their definitions. Valerie provides an example of a participant successfully refining a definition. Her initial written definition for prime was: "A number $x$ is called prime provided the divisors of $x$ are solely 1 and $x$ itself."

I: Is 1 a prime number?

$V: 1$ is not a prime number.

$I:$ Is it by your definition?

$V$ : Yes, because I forgot that part. [She then writes, "An integer $x$ is called prime provided $x>1$ and its divisors are only 1 and $x$ itself."]

I: What about -3 ?

$V$ : It's not a prime because it's not greater than 1 .

I: $\pi$ ?

$V:$ No, because it's not an integer.

I: $\mathrm{OK}, 3$ is divisible by -1 and -3 . So is 3 prime?

$V$ : Based on my definition, no... It wouldn't be a prime.

I: Then are there any prime numbers?

$V$ : I thought so [laughs].

She then amended her definition to include the phrases " $x>1$ " and "it's only positive divisors are only 1 and $x$ itself." From her final definition and from the conversation that lead up to it, we can infer that her concept image of prime number is not only fairly strong (in that she is very confident in it), but we can also see that it is closely aligned with a mathematician's and yet she failed to write a definition that adequately captured her concept image on her first attempt. It was apparent from the conversation, that she had not considered the case of $x=1$, neither had she considered negative integer divisors when writing her initial definition. When these non-examples were supplied to her, she was able to revise her definition to bring it into closer alignment with her concept image. As with trapezoid and prime, the term divisor has alternate definitions. It is sometimes defined in terms of integers, and sometimes defined in terms of natural numbers (and natural numbers are sometimes defined to include zero and sometimes defined to exclude zero). Which definition she accepted was of little interest to us. Instead, we wanted to see if her initial written definition accurately represented her concept image with regard to these key examples.

Heidi's initial definition of even was “\#'s of the form $2 n$ where $n$ is some integer." We asked her if zero was even.

$H$ : Um, I would say zero is even because based on my definition, I have numbers of the form $2 n$ where $n$ is some integer. So, if zero times $2 \ldots$ it would be zero. So, I guess I would say zero is even.

I: And -2 ?

H: Um, -2 is even. Um. Well, I know it's even. I'm not sure, I'm actually not sure if my definition fits with this criteria I guess, but I just know that -2 is even. I just think from the start that we've been prepped to know that $2 n$ is just an even. It means "even number." So, if -2 can be divisible by 2 it gets you -1 . I would say -2 is even.

I: What about $2 \sqrt{3}$ ?

H: I don't think that fits my definition and I honestly don't even know if two-radical-three is even because I don't know what radical three is off the top of my head. So, I'm not sure. So, if I put the number in the form $2 n$ where $n$ is some integer. . .based on my definition, I don't think it fits.

Heidi did not appear to have considered certain key examples and non-examples when writing her definition. She had a very quick response to whether -2 was even which she then supported from her definition, but in the cases of zero and $2 \sqrt{3}$, she went first to her definition to see what it suggested to her. She seems to have recited her definition from memory and then used that written definition along with correct mathematical reasoning to make determinations regarding zero, -2 , and $2 \sqrt{3}$. In so doing, she shows a lot of confidence in her definition and treats it as a stipulative definition and exhibits correct mathematical reasoning by her willingness to accept the consequences of adopting that definition. Valerie also referenced her definition by saying "Based on my definition. . .no [three] wouldn't be prime" and in so doing seems to treat her own definition as stipulative and then recognizes its inadequacy to accurately capture her concept image. Apart from failing to consider key examples, both Valerie and Heidi showed sophisticated reasoning. Both were able refine their definitions to 
bring them into closer alignment with their concept images, and both pointedly defer to their written definitions to see what they revealed about the examples we provided. This suggests an understanding of the prescriptive nature of mathematical definitions.

\subsubsection{Vague concept images}

In cases where participants' concept images were vague or deviated significantly from the widely accepted definitions of these concepts, the interview process sometimes led to some surprising revelations regarding the participant's concept image. Colin's initial written definition of prime was, "Any number not divisible by any number other than 1 and itself." During the interview that followed, Colin revealed something surprising about his concept image of prime number. After testing his definition with examples such as 1 and -3 (both of which he claimed were prime-both by his definition and by his concept image), we asked:

\section{I: What about $\pi$ ?}

C: [It] would have to be a prime number I guess. I mean it's not an integer but I would still say that it would be prime. [Colin rewrites his definition of prime and reads it aloud.] Any number not divisible by an integer other than one and itself. I think that would fix any number not divisible by any other integer other than 1 and itself. I think that would fix it.

I: When you divide $\pi$ by $1 \ldots$

C: You get $\pi$.

I: You get $\pi$. Is $\pi$ an integer?

C: No...so it wouldn't count toward it not being prime.

I: Hold it. . wouldn't count toward it not being. . .

C: I'm sorry! Double negatives...Well, a double negative, you can not NOT be something but it doesn't mean you are something. I don't know, it's all semantics really I guess. . If you divide $\pi$ by itself, you get 1 so it doesn't have to divide by. . . I think I might have to do it by two separate cases. Because if it's an integer, you have to divide it by an integer to get 1 or itself, or any integer. But if it's NOT an integer, then you would have to divide by itself to get 1 and at that point you are dividing by something that is not an integer.

\section{I: What about non-integer numbers?}

C: Um, yeah. I remember going over non-integer numbers and always when it wasn't an integer, it was always just tossed away as prime. I'm pretty sure.

After some probing, it was revealed that a powerful component of Colin's concept image for prime included that if $p$ was a number that couldn't be factored, then $p$ was prime. Numbers that cannot be factored were "tossed away as being prime" and so he ignored the "integer" part of his definition of prime and included non-integers as a way of better capturing his concept image. A reader of Colin's initial written definition could never have adequately understood Colin's concept image, which allowed for non-integers to be prime.

Lynn initially defined an even number to be "An integer that is divisible by 2 with no remainders." We asked:

\section{I: Is zero an even number?}

$L$ :Yes. . Because you don't get anything when. . like zero over 2, I just get zero. I don't get anything else so that's what I mean by no remainders.

\section{I: Are you OK with zero being even?}

L: I wouldn't think it would be odd. You know what I mean? Because like with odd, I feel like. ..with evens it's like being cut in half. Like it can be cut in half evenly. . .So, like negative 2 . . it can be like split up in like two equal parts even though the parts are negative. Does that make sense?

So far, Lynn's answers sound pretty sophisticated. She correctly argued from her definition that zero is an even number, and offered the additional example of $n=-2$ and correctly argued from her definition that -2 could be divided into two equal parts "even though those parts are negative." She explicitly references her definition by saying "that's what I mean by no remainders." However, when discussing -2 , she says "it can be split up into like two equal parts" and no longer alludes to her definition but to an unstated concept image that even numbers can be divided up into equal parts.

Upon further questioning, it became apparent that her concept image of even number opened the door for the inclusion of some non-integers.

I: What about $2 / 4$ ? Is it an even number by your definition?

$L$ : No, it's not. Not by my definition. . .because then I would still have a remainder. . I'm trying to think of a way of why I think one-half is an even number. I think maybe the 2 makes me think that's an even number. 
I: Two fourths or one half. Does one look more even to you than the other?

$L:$ No, I feel they're the same.

I: Two thirds?

$L$ : I would think that was odd because. . I don't know. With one half it like stops. It terminates because it's like point five and then with like two thirds. . would be repeating. OK so that's why I guess I would think like an even number doesn't repeat.

I: So, two fifths? Which is point four?

$L$ : Yeah, I would think that's an even number. Maybe. Yeah, no. . . I feel like I don't like my definition any more. . .I feel like an even number is like a non-repeating [decimal] like it terminates.

From Lynn's initial written definition and her early remarks about zero and -2 , one might have believed that they understood Lynn's concept image for even number. Surprising, latent aspects of her concept image were revealed when she recognized that her initial written definition did not allow for fractional even numbers. She did not ignore her definition as Colin had done but she recognized it was inadequate to capture her concept image and used correct mathematical reasoning to completely abandon her definition in favor of a concept image that allowed certain non-integers (those with terminating decimal expansions) to be in the set of even numbers.

Both Colin's and Lynn's concept images departed from or conflicted with the definitions they gave initially. It appears that initially, both were reciting their definitions from memory but strong components of their concept images had little to do with the definitions they provided. In Colin's case, his concept image included that prime numbers represent a failure at a factoring task. In Lynn's case, her concept image included that even numbers were in some way friendly toward division. Neither of these components were obvious in these participants' initial written definitions and were revealed only after a more careful interrogation.

\subsubsection{Vague concept images of related concepts}

Some of our participants were impeded in writing their definitions when they had difficulties with concepts related to the concept in question. In her definition of even, Sara tried to use language and symbols that sounded mathematical to her by defining, "A number is even provided $2 m=0, m \in \mathbb{R}$." She agreed that zero was an even number and after some discussion regarding her definition which might be interpreted to mean that zero is the only even number, she changed her definition to "A number $x$ is called even provided $2 m=x, m \in \mathbb{R}$." We then asked if $2 \sqrt{3}$ was an even number:

$S$ : Is that one even? . . $\sqrt{3}$ is irrational. So, I don't think it's even. No. I could say it just keeps going. . I have to add like a rational number, a real number. Rational is real?

I: What's the difference between rational and real?

$S$ : Reals is all of them. Oh! Reals is odd numbers too. Because $m$ could be an odd number.

I: Is $\sqrt{3}$ a real number?

$S:$ No, I know it's irrational.

Later, we asked:

I: Is -4 even?

$S$ : Um, reals are all positive. So, no. It's not.

She further amended her definition to read, "A positive rational number $x$ is called even provided $2 m=x, m \in \mathbb{R}$." Rather than dissecting her final definition, we think it is more interesting to draw attention to the difficulties of creating definitions based on poorly understood terms. It is unclear what Sara's concept images were for rational and real numbers. At one point she indicated that "reals is all of them," but later that "reals are all positive." Never did she reveal what she meant by "rational." And further, not once in this exchange did she reference integers. In this she is not alone. Four of our ten participants failed to reference integers in their initial written definitions of even, preferring instead: number, rational number, or real number. Sara wanted "positive rational numbers" that were multiples of 2 to be even.

We wondered if she really wanted to include fractional numbers in the set of even numbers.

I: Is one half an even number?

$S$ : Um, an even number? I think it is an even number.

I: Does it satisfy your definition?

S: Um. I think so. Oh wait! I think so. It is an even number.

I: Why do you say you think it satisfies your definition? 
$S$ : It's positive obviously. And then it's rational because it's like 2 divided by 4 . It stops. So it's rational. It doesn't like go on forever.

I: What about one fifth?

$S:$ Two tenths is one fifth.

I: Does that make it even?

$S$ : I don't think so now.

During the above exchange, Sara became uncertain. She wasn't clear any longer about what she meant when she used the term "even number," and gave up without rewriting her definition.

Tally's initial written definition for even number stated, "Any rational number $n$ that can be written as $2 n$." As with Sara above, a discussion ensued regarding zero and whether any numbers other than zero could be even. She amended her definition to, "Any non-zero rational number $m$ that can be written as $2 n$." Similar to other participants, she did not want zero to be an even number and successfully amended her definition to bring it into closer agreement with her concept image. We then brought up the possibility of fractional numbers.

I: Is two fourths an even number?

$T$ : Yes, because it can be written as 2 times one fourth.

I: How about two thirds then?

T: 2 times one third.

I: OK, how about 1 ?

T: No, it wouldn't work. It would just be 2 over 2 times 1 .

I: Or 2 times 1 over 2 ?

Tally became uncertain.

I: Do you want 1 to be an even number?

T: No.

I: Do you want one half to be an even number?

T: Yes.

I: What about one third?

T: No.

I: But one third is two times one sixth.

T: Oh, man. Isn't one third repeating? So, isn't that, um, now I'm confused about my definition of rational. Rational numbers end. Is that the difference between irrational and rational? Or if there's a pattern then it's...rational. Now I don't like my definition.

I: What do you think of when you think of even numbers?

$T: 2,4,6,8,10$. Multiples of 2 .

Tally abandoned her written definition, yet she could still identify which numbers she really wanted to be even but her concept image somehow allowed for other numbers too. She then gave up trying to reconcile a written definition with her shifting and vague concept image.

In cases where the participants' held weak concept images of not only the concept in question but also of certain related concepts, the participants completely abandoned their initial written definitions when presented with previously unconsidered examples or non-examples. The radical departure from their initial written definitions seems to indicate that the participants did not have a clear concept image from the outset. Further, because of their weak concept images for certain related concepts they may have been reciting or mimicking mathematical language they didn't fully understand when writing definitions. In these cases, as with the previous cases, students' initial written definitions were a poor description of their concept images.

\section{Discussion and conclusion}

Our participants were largely unsuccessful at writing definitions that captured their own concept images on the first attempt. There may be several reasons for this, but it is apparent that they failed to consider key examples when writing 
their definitions. This confirms Moore's (1994) observation that students do not use definitions to generate examples. This seemed to be true even when the definitions in question were their own, regardless of their familiarity with the concept. We were also able to partially confirm Moore's observation that students neither use nor understand mathematical language. All of our participants used mathematical language, but some were apparently attempting to recite definitions and related mathematical language from memory with little understanding.

Consider Heidi, Lynn, Tally, and Sara. Their initial definitions (or early revisions of their definitions) of even number were all similar and at first glance, we might assume they had similar understandings of the concept of even number yet only Heidi's definition would restrict even numbers to integers. Lynn's definition would allow for zero and for all terminating decimals to be even. Sara's would allow zero to be even but not negative integers, and would allow certain non-integers to be even. And Tally would not allow zero to be even but would allow some non-integers even though she could state that what she really wanted was for 2, 4, 6, 8, 10 and other multiples of 2 to be even. Of these, only Heidi mentioned the set of integers in her definition with Sara and Tally preferring the set of rational numbers, and Lynn preferring the set of real numbers although it is by no means clear that these last three really understood the properties of the numbers in these sets and in Lynn's case it is clear that she did not.

Consider Colin and Valerie. Their initial definitions of prime number were nearly identical yet they had very different understandings of what it meant for a number to be prime. The interviews revealed that Valerie wanted positive integers to be prime if they had only two positive integer divisors, while Colin "tossed away" non-integers as being prime because they could not be factored. This difference was not apparent in their initial written definitions.

Because participants wrote and refined their own definitions, their definitions were really extensions of their own concept Images-sometimes poor extensions in which case the definitions were eventually ignored, or abandoned. It is doubtful that any of these participants considered fractions when writing their initial definitions for even number or non-integers when writing their definitions for prime number. A major component of Lynn's, Sara's, and Tally's concept images of even number seemed to be that even numbers were in some way easy to divide into parts. Each were invested in this aspect of their concept images enough to accept the consequences of allowing fractional even numbers. Similarly, a major part of Colin's concept image for prime was a failure at a factoring task and he accepted the consequences of non-integer prime numbers because they satisfied this part of his concept image. In this way, Lynn, Tally, and Colin argued directly from their concept images effectively discarding their own definitions.

Some of the participants never progressed beyond attempting to extract the meaning of prime or even from their concept image. Up until the point that Lynn, Sara, and Tally each abandoned their definitions, they had tried to refine them to better describe what they meant when they used those terms. Valerie and Heidi however, successfully refined their definitions until there came a point that they treated them as stipulative, argued from their definitions rather than from their concept images directly, and accepted the consequences of their definitions. Heidi provides the clearest example when she pointedly references her definition to see what it revealed to her about even numbers.

In the cases presented above, the initial written definitions frequently masked the weak or faulty concept images underlying them. Based only on the initial written definitions, one might have concluded that some of these participants' understandings of the concept of prime or even number were far better than they actually were. It appears that some of these participants had vague notions of what some of these concepts actually meant and in writing definitions that captured their concept images, some attempted to appropriate mathematical language they didn't fully understand. For these participants, their vague concept images of not only the concept in question but especially of related concepts made it difficult for them to extract definitions from their concept images, and to bring their definitions into closer alignment with their concept images.

Ultimately, it is more important to understand a concept than to be able to recite from memory a definition for that concept. In checking for understanding, mathematics teachers at the high school and college levels routinely ask students to provide the definitions of concepts on exams. We find this to be a poor measure of student understanding of the concepts in question. As we have seen above, students can appropriate the language of mathematics with little understanding of that language, and can recite definitions from memory with little understanding of the very concepts they are defining. An ability to provide or recite a definition does not guarantee a robust understanding of the concept in question. Several participants in this study seem to have had vague concept images of basic concepts such as integer, prime number, even number, and real number despite their experiences in advanced college-level mathematics courses (e.g., algebraic structures and college geometry) yet they would likely have been able to conceal this from an instructor who merely asked them for definitions of these or related concepts. For some, this shortcoming only became apparent when they were confronted with key examples and non-examples.

While asking students to define concepts is a poor summative assessment tool, it promises to be a valuable formative assessment tool when combined with follow-up questions, interviews, or other activities. Diagnosing misconceptions, addressing weak concept images, and confronting faulty mathematical behaviors comprise no small part of teaching mathematics. Posing follow-up activities to tasks involving stating definitions can help teachers with each of these aspects of teaching, and may help teachers differentiate instruction for individuals or groups of individuals with similar needs. Learning activities that focus on memorization of definitions address concept images indirectly while carefully planned follow-up activities may reveal latent aspects of students' concept images allowing for a more direct intervention or reification by the teacher.

Our participants were college mathematics majors and had once been successful at high school-level mathematics and were now at least modestly successful at college-level mathematics as evidenced by their progression through the calculus 
sequence, a transition course in mathematical proofs, linear algebra, and into either college geometry or algebraic structures. We conclude that even students who have had significant experience working with definitions and proving theorems may still find it difficult to write definitions that adequately describe their own concept images. Given that the proofs students are asked to provide at the high school and college levels are often relatively straight forward applications of definitions, we are inclined to believe that some of students' difficulties with proofs at these levels are symptoms of students' difficulties with definitions.

\section{References}

Dickerson, D., \& Pitman, D. (2012). Advanced college-level students' categorization and use of mathematical definitions. In T. Y. Tso (Ed.), Proceedings of the 36 th conference of the international group or the psychology of mathematics education (pp. 187-193).

Edwards, B. S., \& Ward, M. B. (2004). Surprises from mathematics education research: Student (mis)use of mathematical definitions. The American Mathematical Monthly, 111(5), 411-424.

Goldin, G. (2000). A scientific perspective on structured, task-based interviews in mathematics education research. In A. Kelley, \& R. Lesh (Eds.), Handbook of research design in mathematics and science education (pp. 517-545). Mahwah, NJ: Lawrence Erlbaum Associates, Publishers.

Harel, G., \& Sowder, L. (1998). Students' proof schemes: Results from exploratory studies. In A. Schoenfeld, J. Kaput, \& E. Dubinsky (Eds.), Research in collegiate mathematics education, III (pp. 234-283). Washington, DC: Mathematical Association of America.

Moore, R. (1994). Making the transition to formal proof. Educational Studies in Mathematics, 27, 249-266.

Tall, D., \& Vinner, S. (1981). Concept image and concept definition in mathematics with particular reference to limits and continuity. Educational Studies in Mathematics, 12(2), 151-169.

Vinner, S. (1977). The concept of exponentiation at the undergraduate level and the definitional approach. Educational Studies in Mathematics, 8(1), $151-169$.

Vinner, S. (1991). The role of definitions in the teaching and learning of mathematics. In D. Tall (Ed.), Advanced mathematical thinking (pp. 65-81). Kluwer: Dordrecht, The Netherlands.

Wilson, P. (1990). Inconsistent ideas related to definitions and examples. Focus on Learning Problems in Mathematics, 12(3\&4), 31-47.

Wheeler, A., \& Champion, J. (2013). Students' proofs of one-to-one and onto properties in introductory abstract algebra. International Journal of Mathematical Education in Science and Technology, 44(8), 1107-1116. 